\title{
MULTIPLICITIES OF HIGHER LIE CHARACTERS
}

\author{
MANFRED SCHOCKER
}

(Received 17 June 2001; revised 26 April 2002)

Communicated by Jie $\mathrm{Du}$

\begin{abstract}
The higher Lie characters of the symmetric group $S_{n}$ arise from the Poincare-Birkhoff-Witt basis of the free associative algebra. They are indexed by the partitions of $n$ and sum up to the regular character of $S_{n}$. A combinatorial description of the multiplicities of their irreducible components is given. As a special case the Kraśkiewicz-Weyman result on the multiplicities of the classical Lie character is obtained.
\end{abstract}

2000 Mathematics subject classification: primary 20C30*, 05E10, $17 \mathrm{~B} 99$.

Keywords and phrases: symmetric group, general linear group, free Lie algebra, tableau, major index.

\section{Introduction}

At the beginning of the last century Schur studied the structure of the tensor algebra $T(V)$ over a finite dimensional $K$-vector space $V$ as a $G L(V)$-module. In his thesis ([13]) and a famous subsequent paper ([14]) he was able to describe the decomposition of the homogeneous components

$$
T_{n}(V):=\underbrace{V \otimes \cdots \otimes V}_{n}
$$

of degree $n$ in $T(V)$ into irreducible GL(V)-modules using the irreducible representations of the symmetric group $S_{n}$. The usual Lie bracketing $[x, y]:=x y-y x$ turns $T(V)$ into a Lie algebra. The Lie subalgebra $L(V)$ generated by $V$ is free over any basis of $V$ by a classical result of Witt ([17]), and $L_{n}(V):=T_{n}(V) \cap L(V)$ is a GL(V)-submodule of $T_{n}(V)$ for all $n$. Let $q=q_{1} \ldots q_{k}$ be a partition of $n$, that is, $q_{1} \geq \cdots \geq q_{k}$ and $q_{1}+\cdots+q_{k}=n$. Then we define

$$
\left.L_{q}(V):=\left\langle\sum_{\pi \in S_{k}} P_{1 \pi} \cdots P_{k \pi}\right| P_{i} \in L_{q_{i}}(V) \text { for } 1 \leq i \leq k\right\rangle_{K} .
$$

(C) 2003 Australian Mathematical Society $1446-7887 / 03 \$ A 2.00+0.00$ 
By the Poincaré-Birkhoff-Witt theorem, $T_{n}(V)$ is the direct sum of these subspaces:

$$
T_{n}(V)=\bigoplus_{q \vdash n} L_{q}(V),
$$

and this decomposition is $\mathrm{GL}(V)$-invariant.

Meanwhile, different families of idempotents $e_{q}$ in the group algebra $K S_{n}$ indexed by partitions have been introduced such that $L_{q}(V) \cong e_{q} T_{n}(V)$ for all $q$ (see, for example, $[2,3,11])$. For any decomposition $e_{q} K S_{n}=\bigoplus_{p} a_{q, p} M_{p}$ into irreducible $S_{n}$-modules, we now have

$$
L_{q}(V)=e_{q} T_{n}(V) \cong e_{q} K S_{n} \otimes_{K S_{n}} T_{n}(V)=\bigoplus_{p} a_{q, p}\left(M_{p} \otimes_{K S_{n}} T_{n}(V)\right) .
$$

In this decomposition, by Schur's fundamental result, $M_{p} \otimes_{K S_{n}} T_{n}(V)$ is either 0 or an irreducible GL(V)-module. Hence the GL(V)-module structure of $L_{q}(V)$ is completely determined by the multiplicities $a_{q, p}$ of the higher Lie module $e_{q} K S_{n}$ of $S_{n}$. In this vein, for the special case of $q=n$, the problem of describing the $\mathrm{GL}(V)$-module structure of $L_{n}(V)$ formulated by Thrall ([16]) could finally be solved in a satisfying way by works of Klyachko ([8]) and Kraśkiewicz and Weyman ([9]).

The higher Lie characters $\lambda_{q}$ of $S_{n}$ corresponding to the modules $e_{q} K S_{n}$ sum up to the regular character of $S_{n}$, by (1), and it is natural to ask for their multiplicities for arbitrary $q$. In this paper, a combinatorial description of these multiplicities is given in terms of alternating sums of numbers of standard tableaux with certain major index properties (Section 3). For $q=n$, we obtain the Kraśkiewicz-Weyman result mentioned above. Our approach is based on a generalization of Klyachko's result (Section 2) combined with the calculus of noncommutative character theory introduced in [6] (Section 4).

\section{The reduction to partitions of block type}

Let $q$ be a partition of $n$. The higher Lie character $\lambda_{q}$ is induced by a certain linear character of the centralizer of an element of cycle type $q$ in $S_{n}$. For $q=n$, this result is due to Klyachko ([8]). In full generality, it is implicitly contained in [1] for the first time (for details, see [12, Section 8.5]) and will be briefly recalled in two steps in this section.

Let $\mathbb{N}\left(\mathbb{N}_{0}\right.$, respectively) be the set of all positive (nonnegative, respectively) integers and $n_{1}:=\{k \in \mathbb{N} \mid k \leq n\}$ for all $n \in \mathbb{N}_{0}$. Let $\mathbb{N}^{*}$ be a free monoid over the alphabet $\mathbb{N}$. We write $q . r$ for the concatenation product of $q, r \in \mathbb{N}^{*}$ in order to avoid confusion with the ordinary product in $\mathbb{N}$. Accordingly, we denote by $d^{k}$ the $k$-th power of a letter $d \in \mathbb{N}$ in $\mathbb{N}^{*}$, for all $k \in \mathbb{N}_{0}$. If $n \in \mathbb{N}$ and $q=q_{1} \ldots q_{k} \in \mathbb{N}^{*}$ such that 
$q_{1}+\cdots+q_{k}=n$, we say that $q$ is a composition of $n$ of length $|q|:=k$, and write $q \models n$. If, additionally, $q_{1} \geq \cdots \geq q_{k}$ and hence $q$ is a partition of $n$, we write $q \vdash n$.

Let $K$ be a field of characteristic 0 containing a primitive $n$-th root of unity $\varepsilon_{n}$ for all $n \in \mathbb{N}$. For all $n \in \mathbb{N}_{0}$, we denote by $\mathrm{Cl}_{K}\left(S_{n}\right)$ the ring of class functions of the symmetric group $S_{n}$. Let $C_{q}$ be the conjugacy class consisting of all permutations $\pi$ whose cycle partition $z(\pi)$ is a rearrangement of $q$, for all $q \in \mathbb{N}^{*}$. Let $\operatorname{ch}_{q} \in \mathrm{Cl}_{K}\left(S_{n}\right)$ such that $\left(\chi, \mathrm{ch}_{q}\right)_{S_{n}}=\chi\left(C_{q}\right)$ is the value of $\chi$ on any element $\pi \in C_{q}$ for all $\chi \in \mathrm{Cl}_{K}\left(S_{n}\right)$. Then, up to a certain factor, $\mathrm{ch}_{q}$ is the characteristic function of $C_{q}$ in $\mathrm{Cl}_{K}\left(S_{n}\right)$, and we have $C_{q}=C_{r}$ and $\mathrm{ch}_{q}=\mathrm{ch}_{r}$ whenever $q$ is a rearrangement of $r$, for all $q, r \in \mathbb{N}^{*}$. The outer product $\bullet$ on the direct sum $\mathrm{Cl}:=\bigoplus_{n \in \mathbb{N}_{0}} \mathrm{Cl}_{K}\left(S_{n}\right)$ may now be defined by

$$
\operatorname{ch}_{q} \bullet \mathrm{ch}_{r}:=\operatorname{ch}_{q . r}
$$

for all $q, r \in \mathbb{N}^{*}$. It corresponds via Frobenius' characteristic mapping to the ordinary multiplication of symmetric functions.

Our starting point is the following part of [12, Theorem 8.23], which already occurs in $[16$, Section 8$]$.

Lemma 2.1. Let $n \in \mathbb{N}$ and $q \vdash n$. Denote by $a_{i}$ the multiplicity of the letter $i$ in $q$, for all $i \in \underline{n}$. Then we have $\lambda_{q}=\lambda_{n^{\cdot a_{n}}} \bullet \cdots \cdot \lambda_{1^{\cdot a_{1}}}$.

Hence, with $\zeta^{p}$ denoting the irreducible character of $S_{n}$ corresponding to $p$ for $p \vdash n$, the problem of describing the multiplicities

$$
a_{q, p}:=\left(\lambda_{q}, \zeta^{p}\right)_{S_{n}}
$$

may be reduced to the case that $q$ is of block type, that is, $q=d^{k}$ is the $k$-th power of a single letter $d$. Indeed, for partitions $q=q_{1} \ldots q_{k} \vdash x, r=r_{1} \ldots \ldots r_{l} \vdash y$ such that $q_{k}>r_{1}$ and $x+y=n$, we have

$$
\left(\lambda_{q . r}, \zeta^{p}\right)_{s_{n}}=\left(\lambda_{q} \bullet \lambda_{r}, \zeta^{p}\right)_{s_{n}}=\sum_{s \vdash x} \sum_{t \vdash y} c_{s, t}^{p} a_{q, s} a_{r, t}
$$

by Lemma 2.1 , where $c_{s, t}^{p}=\left(\zeta^{s} \bullet \zeta^{t}, \zeta^{p}\right)_{s_{n}}$ is the well-known Littlewood-Richardson coefficient.

For all $n, m \in \mathbb{N}_{0}, \psi \in S_{n}$ and $\sigma \in S_{m}$, we define $\psi \# \sigma \in S_{n+m}$ by

$$
i(\psi \# \sigma):= \begin{cases}i \psi & i \leq n ; \\ (i-n) \sigma+n & i>n\end{cases}
$$

for all $i \in \underline{n+m}$. Furthermore, for $d, k \in \mathbb{N}, n:=d k$ and $\pi \in S_{k}$, we define $\pi^{\left[d^{k}\right]} \in S_{n}$ by

$$
(d j-i) \pi^{\left[d^{\cdot k}\right]}:=d(j \pi)-i
$$


for all $j \in \underline{k}, i \in \underline{d-1} \cup\{0\}$. That is, $\pi^{\left[d^{k}\right]}$ is permuting the $k$ successive blocks of length $d$ in $n$ according to $\pi$. Now let $\tau_{d}:=(1, \ldots, d) \in S_{d}$ be the standard cycle of length $d$ in $S_{d}$ and put

$$
\sigma_{d^{k}}:=\underbrace{\tau_{d} \# \cdots \# \tau_{d}}_{k} \in C_{d^{k}} \subseteq S_{n} .
$$

Then the centralizer of $\sigma_{d^{k}}$ in $S_{n}$ is a wreath product of the cyclic group generated by $\tau_{d}$ with $S_{k}$ and may be described as

$$
C^{d^{k}}:=C_{S_{n}}\left(\sigma_{d^{k}}\right)=\left\{\pi^{\left[d^{k}\right]}\left(\tau_{d}^{i_{1}} \# \cdots \# \tau_{d}^{i_{k}}\right) \mid \pi \in S_{k}, i_{1}, \ldots, i_{k} \in \underline{d}\right\} .
$$

([5, Section 4.1]). With these notations, the remaining part of Theorem 8.23 in [12], transferred to $\mathrm{Cl}$, reads as follows.

THEOREM 2.2. Let $d, k \in \mathbb{N}$ and $n:=d k$. Then

$$
\psi_{d^{k}}: C^{d^{k}} \longrightarrow K, \quad \pi^{\left[d^{k}\right]}\left(\tau_{d}^{i_{1}} \# \cdots \# \tau_{d}^{i_{k}}\right) \longmapsto \varepsilon_{d}^{-\left(i_{1}+\cdots+i_{k}\right)}
$$

is a linear representation of $C^{d^{k}}$, and $\left(\psi_{d^{\cdot k}}\right)^{S_{n}}=\lambda_{d^{\cdot k}}$.

\section{Multiplicities}

In order to state our main result (Theorem 3.1), we need the notion of a standard Young tableau and its multi major index corresponding to a composition. Let $n \in \mathbb{N}$ and $p=p_{1} \ldots p_{l} \vdash n$. The frame $R(p):=\left\{(i, j) \in \mathbb{N} \times \mathbb{N} \mid i \in l, j \in \underline{p_{i j}}\right\}$ corresponding to $p$ may be visualized by its Ferrers diagram, an array of boxes with $p_{1}$ boxes in the first (top) row, $p_{2}$ boxes in the second row and so on. For example, we have

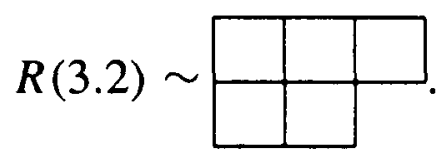

The images $1 \pi, \ldots, n \pi$ of any permutation $\pi \in S_{n}$ may be entered into $R(p)$ row by row, starting at bottom left and ending at top right. Let $\mathrm{SYT}^{p}$ be the set of all permutations which are increasing in rows (from left to right) and columns (downwards) when entered into $R(p)$ in this way. The elements of SYT ${ }^{p}$ are called standard Young tableaux of shape $p$. In the above example, the elements of SYT ${ }^{3.2}$, entered into $R(3.2)$, are

\begin{tabular}{|l|l|l|}
\hline 1 & 2 & 3 \\
\hline 4 & 5 & \\
\hline
\end{tabular}
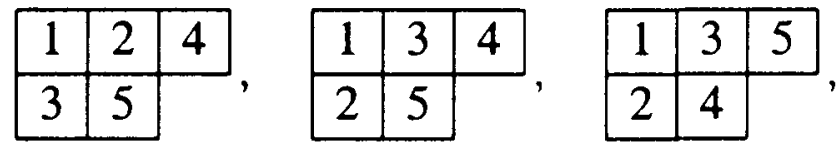

\begin{tabular}{|l|l|l|}
\hline 1 & 2 & 5 \\
\hline 3 & 4 & \\
\hline
\end{tabular}


Accordingly, we obtain

$$
\mathrm{SYT}^{3.2}=\left\{\left(\begin{array}{l}
12345 \\
45123
\end{array}\right),\left(\begin{array}{l}
12345 \\
35124
\end{array}\right),\left(\begin{array}{l}
12345 \\
25134
\end{array}\right),\left(\begin{array}{l}
12345 \\
24135
\end{array}\right),\left(\begin{array}{l}
12345 \\
34125
\end{array}\right)\right\} \subseteq S_{5} .
$$

For all $\pi \in S_{n}, D(\pi):=\{i \in \underline{n-1} \mid i \pi>(i+1) \pi\}$ is called the descent set of $\pi$. Let $q=q_{1} \ldots q_{k} \vDash n$ and put $s_{j}:=q_{1}+\cdots+q_{j}$ for all $j \in \underline{k} \cup\{0\}$. Then the multi major index of $\pi$ corresponding to $q$ is defined as

$$
\operatorname{maj}_{q} \pi:=m_{1} \ldots . m_{k} \in \mathbb{N}^{*}
$$

where

$$
m_{j}:=\sum_{\substack{s_{j-1}<i \leq s_{j} \\ i \in D(\pi)}}\left(i-s_{j-1}\right)
$$

for all $j \in \underline{k}$. For $q=n$, we obtain the ordinary major index maj $\pi:=\operatorname{maj}_{n} \pi$ of $\pi$. If, additionally, $r=r_{1} \ldots r_{k} \in \mathbb{N}^{*}$, we define

$$
\operatorname{syt}_{q, r}^{p}:=\left|\left\{\pi \in \operatorname{SYT}^{p} \mid \forall j \in \underline{k}_{1}:\left(\operatorname{maj}_{q}\left(\pi^{-1}\right)\right)_{j} \equiv r_{j} \quad \bmod q_{j}\right\}\right| .
$$

Here $\left(\operatorname{maj}_{q}\left(\pi^{-1}\right)\right)_{j}$ always denotes the $j$-th letter of $\operatorname{maj}_{q}\left(\pi^{-1}\right)$, for all $j \in \underline{k}$. For arbitrary $r=r_{1} \ldots . r_{l}, q=q_{1} \ldots q_{k} \in \mathbb{N}^{*}$ we write $r \mid q$ if and only if $l=k$ and $r_{i}$ is a divisor of $q_{i}$ for all $i \in k_{\text {s. }}$. In this case, we define furthermore the following extension of the number theoretic Möbius function $\mu$ :

$$
\mu(q / r):=\prod_{i=1}^{|q|} \mu\left(q_{i} / r_{i}\right)
$$

Finally, for $k \in \mathbb{N}$ and $r=r_{1} \ldots . r_{l} \in \mathbb{N}^{*}$, we put $k \star r:=\left(k r_{1}\right) \ldots .\left(k r_{l}\right)$.

MAIN THEOREM 3.1. Let $d, k, n \in \mathbb{N}$ such that $d k=n$. Let $p \vdash n$. Then we have

$$
\left(\lambda_{d^{k}}, \zeta^{p}\right)_{S_{n}}=\frac{1}{k !} \sum_{q \vdash k}\left|C_{q}\right| \sum_{r \mid q} \mu(q / r) \operatorname{syt}_{d \star q, r}^{p} .
$$

The proof will be given in Section 5. A description of the multiplicity $\left(\lambda_{q}, \zeta^{p}\right)_{S_{n}}$ for arbitrary $q \vdash n$ may be obtained from Theorem 3.1 via (3). For $k \leq 3$, we obtain the following specializations of Theorem 3.1, the first of which is due to Kraśkiewicz and Weyman (see the Remark at the end of this section).

COROLlary 3.2. Let $d \in \mathbb{N}$.

(a) For all $p \vdash d$, we have $\left(\lambda_{d}, \zeta^{p}\right)_{S_{d}}=\operatorname{syt}_{d, 1}^{p}$.

(b) For all $p \vdash 2 d$, we have $\left(\lambda_{d . d}, \zeta^{p}\right)_{s_{2 d}}=1 / 2\left(\operatorname{syt}_{d \cdot d, 1.1}^{p}+\operatorname{syt}_{2 d, 2}^{p}-\operatorname{syt}_{2 d, 1}^{p}\right)$. 
TABLE 1.

\begin{tabular}{|c|c|c|c|c|c|c|}
\hline \multicolumn{2}{|c|}{$\pi$} & $\pi^{-1}$ & $\mathrm{maj}_{6} \pi^{-1}$ & $\operatorname{maj}_{3.3} \pi^{-1}$ & $\operatorname{maj}_{2.2 .2} \pi^{-1}$ & $\mathrm{maj}_{4.2} \pi^{-1}$ \\
\hline 1 & $\underline{2}$ & \multirow{3}{*}{$\left(\begin{array}{l}123456 \\
563412\end{array}\right)$} & \multirow{3}{*}{6} & \multirow{3}{*}{2.1} & \multirow{3}{*}{0.0 .0} & \multirow{3}{*}{2.0} \\
\hline 3 & $\underline{4}$ & & & & & \\
\hline 5 & 6 & & & & & \\
\hline 1 & $\underline{2}$ & \multirow{3}{*}{$\left(\begin{array}{l}123456 \\
563142\end{array}\right)$} & \multirow{3}{*}{10} & \multirow{3}{*}{2.2} & \multirow{3}{*}{0.1 .1} & \multirow{3}{*}{5.1} \\
\hline$\underline{3}$ & 5 & & & & & \\
\hline 4 & 6 & & & & & \\
\hline$\underline{1}$ & $\underline{3}$ & \multirow{3}{*}{$\left(\begin{array}{l}123456 \\
536412\end{array}\right)$} & \multirow{3}{*}{8} & \multirow{3}{*}{1.1} & \multirow{3}{*}{1.1 .0} & \multirow{3}{*}{4.0} \\
\hline 2 & 4 & & & & & \\
\hline 5 & 6 & & & & & \\
\hline$\underline{1}$ & $\underline{3}$ & \multirow{3}{*}{$\left(\begin{array}{l}123456 \\
536142\end{array}\right)$} & \multirow{3}{*}{9} & \multirow{3}{*}{1.2} & \multirow{3}{*}{1.1 .1} & \multirow{3}{*}{4.1} \\
\hline 2 & $\underline{5}$ & & & & & \\
\hline 4 & 6 & & & & & \\
\hline 1 & 4 & \multirow{3}{*}{$\left(\begin{array}{l}123456 \\
531642\end{array}\right)$} & \multirow{3}{*}{12} & \multirow{3}{*}{3.3} & \multirow{3}{*}{1.0 .1} & \multirow{3}{*}{3.1} \\
\hline 2 & $\underline{5}$ & & & & & \\
\hline 3 & 6 & & & & & \\
\hline
\end{tabular}

(c) For all $p \vdash 3 d$, we have

$\left(\lambda_{d . d . d}, \zeta^{p}\right)_{S_{3 d}}=\frac{1}{6}\left(\operatorname{syt}_{d . d . d .1 .1 .1}^{p}+3\left(\operatorname{syt}_{(2 d) . d, 2.1}^{p}-\operatorname{syt}_{(2 d) . d, 1.1}^{p}\right)+2\left(\operatorname{syt}_{3 d, 3}^{p}-\operatorname{syt}_{3 d, 1}^{p}\right)\right)$.

We will illustrate Corollary 3.2 in the case of $p=2.2 .2$. The standard Young tableaux $\pi$ of shape $p$ are listed in Table 1 together with their multi major indices in question. The descents of $\pi^{-1}$ are underlined in each case.

By Corollary 3.2, we obtain $\left(\lambda_{6}, \zeta^{2.2 .2}\right)_{S_{6}}=0$ and furthermore

$$
\left(\lambda_{3.3}, \zeta^{2.2 .2}\right)_{S_{6}}=\frac{1}{2}(1+1-0)=1
$$

and

$$
\left(\lambda_{2.2 .2}, \zeta^{2.2 .2}\right)_{S_{6}}=\frac{1}{6}(1+3(0-1)+2(1-0))=0 .
$$

For $p \vdash d \in \mathbb{N}$ and $\pi \in \mathrm{SYT}^{p}$, note that $i \in d-1$ is a descent of $\pi^{-1}$ if and only if $i$ stands strictly above $i+1$ in $\pi$, entered into $R(p)$. Hence Corollary 3.2 (a) indeed coincides with the original result of Kraśkiewicz and Weyman on the Lie character $\lambda_{d}([9])$. 


\section{Noncommutative character theory}

Let $n \in \mathbb{N}$. The descent algebra $\mathscr{D}_{n}$ is defined as the linear span of the elements $\delta^{D}:=\sum\left\{\pi \in S_{n} \mid D(\pi)=D\right\}\left(D \subseteq n-1_{1}\right)$ in $K S_{n}$. Due to Solomon ([15]), $\mathscr{D}_{n}$ is a subalgebra of $K S_{n}$, and there exists a certain epimorphism of algebras $c_{n}: \mathscr{D}_{n} \rightarrow$ $\mathrm{Cl}_{K}\left(S_{n}\right)$, for all $n$. The direct sum $K S:=\bigoplus_{n \in \mathbb{N}} K S_{n}$ is a graded algebra with respect to the convolution product $\bullet$ (see $[6,1.3]$ for a combinatorial description), and $\mathscr{D}:=\bigoplus_{n \in \mathbb{N}} \mathscr{D}_{n}$ is a $\bullet$-subalgebra of $K S$ (see [12]). In [6], a (noncommutative) --subalgebra $\mathscr{R}$ of $K S$ and a $\bullet$-homomorphism $c: \mathscr{R} \rightarrow \mathrm{Cl}$ are introduced such that $\mathscr{D} \subseteq \mathscr{R}$ and $\left.c\right|_{\mathscr{D}_{n}}=c_{n}$ for all $n$. Furthermore, a (bilinear) scalar product $(\cdot, \cdot)$ on $K S$ is defined by

$$
(\pi, \sigma):= \begin{cases}1 & \pi=\sigma^{-1} \\ 0 & \pi \neq \sigma^{-1}\end{cases}
$$

for all permutations $\pi, \sigma$, and it is shown that

$$
(\varphi, \psi)=(c(\varphi), c(\psi))_{s}
$$

for all $\varphi, \psi \in \mathscr{R}$, where the scalar product on the right hand side is the canonical orthogonal extension of the ordinary scalar products $(\cdot, \cdot)_{S_{n}}$ on $\mathrm{Cl}_{K}\left(S_{n}\right), n \in \mathbb{N}$. For any partition $p \in \mathbb{N}^{*}, Z^{p}:=\sum_{\pi \in S Y T^{p}} \pi$ is an element of $\mathscr{R}$ such that

$$
c\left(Z^{p}\right)=\zeta^{p}
$$

is the irreducible character of $S_{n}$ corresponding to $p$. For example, for $p=3.2$, we obtain $Z^{3.2}=\left(\begin{array}{l}12345 \\ 45123\end{array}\right)+\left(\begin{array}{l}12345 \\ 35124\end{array}\right)+\left(\begin{array}{l}12345 \\ 25134\end{array}\right)+\left(\begin{array}{l}12345 \\ 24135\end{array}\right)+\left(\begin{array}{l}12345 \\ 34125\end{array}\right)$. These results provide the following general concept for describing multiplicities: Given an arbitrary character $\chi \in \mathrm{Cl}_{K}\left(S_{n}\right)$, any inverse image $\varphi \in \mathscr{R}$ of $\chi$ under $c$ may be understood as a noncommutative character corresponding to $\chi$. By (8) and (9), for each such $\varphi$, it follows that

$$
\left(\chi, \zeta^{p}\right)_{S_{n}}=\left(c(\varphi), c\left(Z^{p}\right)\right)_{S_{n}}=\left(\varphi, Z^{p}\right) .
$$

The right-hand side of (10) gives different combinatorial descriptions of the multiplicity on the left-hand side, according to the choice of $\varphi$, simply by the definition of $Z^{p}$ and the scalar product on $\mathscr{R}$.

\section{Klyachkos's idempotent and Ramanujan sums}

In the sequel, following the concept described in Section 4, an inverse image of $\lambda_{d^{*}}$ under $c$ in $\mathscr{D}$ is constructed. It leads to a short proof of our main result Theorem 3.1, by means of (10). 
Let $n \in \mathbb{N}$. We put $\kappa_{n}(x):=\sum_{\pi \in S_{n}} x^{\text {maj } \pi} \pi(x$ a variable $)$ and

$$
M_{n, i}:=\sum_{\substack{\pi \in S_{n} \\ \operatorname{maj} \equiv i \bmod n}} \pi \in \mathscr{D}_{n}
$$

for all $i \in \mathbb{N}_{0}$. Then, up to the factor $1 / n, \kappa_{n}\left(\varepsilon_{n}\right)=\sum_{i=1}^{n} \varepsilon_{n}^{i} M_{n, i} \in \mathscr{D}_{n}$ is a Lie idempotent, that is, $\kappa_{n}^{2}=n \kappa_{n}$ and $L_{n}(V)=\kappa_{n} T_{n}(V)$. This remarkable result is due to Klyachko ([8]).

LEMMA 5.1. Let $n, i \in \mathbb{N}$ and $d$ be the order of $\varepsilon_{n}^{i}$. Then we have

$$
\kappa_{n}\left(\varepsilon_{n}^{i}\right)=\underbrace{\kappa_{d}\left(\varepsilon_{n}^{i}\right) \bullet \cdots \bullet \kappa_{d}\left(\varepsilon_{n}^{i}\right)}_{n / d} .
$$

In particular, $c\left(\kappa_{n}\left(\varepsilon_{n}^{i}\right)\right)=\operatorname{ch}_{d^{n / d}}$.

The main part of the preceding lemma is a special case of [10, Proposition 4.1], while the additional claim on the $c$-image follows from [7, Proposition 1]. For $n, m \in \mathbb{N}$, we denote by $\operatorname{gcd}(n, m)$ the greatest common divisor of $n$ and $m$.

COROLlaRY 5.2. Let $n \in \mathbb{N}$ and $i, j \in \mathbb{N}_{0}$ such that $\operatorname{gcd}(i, n)=\operatorname{gcd}(j, n)$. Then $c\left(M_{n, i}\right)=c\left(M_{n, j}\right)$.

ProOF. As $\operatorname{gcd}(i, n)=\operatorname{gcd}(j, n)$, we can find an integer $m \in \mathbb{N}$ such that $i \equiv j m$ modulo $n$ and $\operatorname{gcd}(m, n)=1$. For all $k \in \mathbb{N}$, we have $\operatorname{gcd}(k m, n)=\operatorname{gcd}(k, n)$ and hence $c\left(\kappa_{n}\left(\varepsilon_{n}^{k}\right)\right)=c\left(\kappa_{n}\left(\varepsilon_{n}^{m k}\right)\right)$, by Lemma 5.1. It follows that

$$
\begin{aligned}
n c\left(M_{n, i}\right) & =c\left(\sum_{l=1}^{n} \sum_{k=1}^{n}\left(\varepsilon_{n}^{l-i}\right)^{k} M_{n, l}\right)=c\left(\sum_{k=1}^{n} \varepsilon_{n}^{-i k} \kappa_{n}\left(\varepsilon_{n}^{k}\right)\right) \\
& =c\left(\sum_{k=1}^{n} \varepsilon_{n}^{-i k} \kappa_{n}\left(\varepsilon_{n}^{m k}\right)\right)=c\left(\sum_{l=1}^{n} \sum_{k=1}^{n}\left(\varepsilon_{n}^{l m-i}\right)^{k} M_{n, l}\right) \\
& =c\left(\sum_{l=1}^{n} \sum_{k=1}^{n}\left(\left(\varepsilon_{n}^{m}\right)^{l-j}\right)^{k} M_{n, l}\right)=n c\left(M_{n, j}\right) .
\end{aligned}
$$

Let $n, m \in \mathbb{N}$. The Ramanujan sum corresponding to $n$ and $m$ is defined by

$$
\varrho(n, m):=\sum \varepsilon^{m}
$$

where the sum is taken over all primitive $n$-th roots of unity $\varepsilon$. In the particular case of $m=1$ ( $m=n$, respectively), $\varrho(n, m)$ yields the Möbius function $\mu(n)=\varrho(n, 1)$ 
(Euler's function $\varphi(n)=\varrho(n, n)$, respectively). We write $x \mid m$, if $x \in \mathbb{N}$ is a divisor of $m$, and put

$$
R(n, m):=\sum_{x \mid m} \varrho(n, x) \varrho(m / x, 1)
$$

Now, for all $d, k \in \mathbb{N}$ and $p=p_{1} \ldots . p_{l} \in \mathbb{N}^{*}$, let

$$
M_{d}(k):=\sum_{y \mid d k} R(d k / y, d) M_{d k, y}
$$

and

$$
M_{d}(p):=M_{d}\left(p_{1}\right) \bullet \cdots \bullet M_{d}\left(p_{l}\right)
$$

Note that $M_{d}(p) \in \mathscr{D}$, as $\mathscr{D}$ is closed under the convolution product.

LEMMA 5.3. For all $d, k \in \mathbb{N}$, we have

$$
\lambda_{d^{k}}=c\left(\frac{1}{k !} \sum_{\pi \in S_{k}} \frac{1}{d^{|z(\pi)|}} M_{d}(z(\pi))\right) .
$$

(Recall that $z(\pi)$ denotes the cycle partition of $\pi$ for any permutation $\pi$.)

PROOF. We write

$$
z\left(\pi ; i_{1}, \ldots, i_{k}\right):=z\left(\pi^{\left[d^{\cdot k}\right]}\left(\tau_{d}^{i_{1}} \# \cdots \# \tau_{d}^{i_{k}}\right)\right)
$$

for all $\pi \in S_{k}, i_{1}, \ldots, i_{k} \in d-1 \cup\{0\}$. By Theorem 2.2, we then have

$$
\begin{aligned}
\lambda_{d^{\cdot k}} & =\frac{1}{\left|C^{d^{k}}\right|} \sum_{q 1-d k}\left(\sum_{\substack{\varphi \in C^{d^{k}} \\
z(\varphi)=q}} \psi_{d^{\cdot k}}(\varphi)\right) \operatorname{ch}_{q} \\
& =\frac{1}{k !} \sum_{\pi \in S_{k}} \frac{1}{d^{k}} \sum_{i_{1}, \ldots, i_{k}=0}^{d-1} \varepsilon_{d}^{-\sum i_{j}} \operatorname{ch}_{z\left(\pi ; i_{1}, \ldots, i_{k}\right)} .
\end{aligned}
$$

By induction on the number $z=|z(\pi)|$ of cycles in $\pi \in S_{k}$, we show that

$$
\frac{1}{d^{k}} \sum_{i_{1}, \ldots, i_{k}=0}^{d-1} \varepsilon_{d}^{-\sum i_{j}} \operatorname{ch}_{z\left(\pi ; i_{1}, \ldots, i_{k}\right)}=c\left(\frac{1}{d^{z}} M_{d}(z(\pi))\right),
$$

which implies our claim. We will use some basic facts about cycle partitions of elements of $C^{d^{. k}}$ which can be found in [5, 4.2]. Let $z=1$. Then $\pi \in S_{k}$ is a long 
cycle. Putting $\eta:=\varepsilon_{k d}$ and applying [5, 4.2.17], Lemma 5.1 and Corollary 5.2, we obtain

$$
\begin{aligned}
& \frac{1}{d^{k}} \sum_{i_{1}, \ldots, i_{k}=0}^{d-1} \varepsilon_{d}^{-\sum i_{j}} \operatorname{ch}_{z\left(\pi ; i_{1}, \ldots, i_{k}\right)} \\
& =\frac{1}{d} \sum_{i=0}^{d-1} \varepsilon_{d}^{-i} \operatorname{ch}_{k * z\left(\tau_{d}^{j}\right)}=\frac{1}{d} \sum_{x \mid d} \varrho(d / x, 1) \operatorname{ch}_{k * z\left(\tau_{d}^{x}\right)} \\
& =c\left(\frac{1}{d} \sum_{x \mid d} \varrho(d / x, 1) \kappa_{k d}\left(\eta^{x}\right)\right)=c\left(\frac{1}{d} \sum_{x \mid d} \sum_{j=0}^{d k-1} \varrho(d / x, 1) \eta^{j x} M_{d k}^{(j)}\right) \\
& =c\left(\frac{1}{d} \sum_{y \mid d k} M_{d k}^{(y)} \sum_{x \mid d} \varrho(d / x, 1) \varrho(d k / y, x)\right)=c\left(\frac{1}{d} \sum_{y \mid d k} M_{d k}^{(y)} R(d k / y, d)\right) \\
& =c\left(M_{d}(k) / d\right) .
\end{aligned}
$$

Now let $z>1$, say, $\pi=\tilde{\pi} \sigma$ for a cycle $\sigma$ of length $l$ in $\pi$. Then we have, by [5, 4.2.19], (2) and our induction hypothesis,

$$
\begin{aligned}
& \frac{1}{d^{k}} \sum_{i_{1}, \ldots, i_{k}=0}^{d-1} \varepsilon_{d}^{-\sum i_{j}} \operatorname{ch}_{z\left(\pi ; i_{1}, \ldots, i_{k}\right)} \\
& =\left(\frac{1}{d^{k-l}} \sum_{i_{1}, \ldots, i_{k-l}=0}^{d-1} \varepsilon_{d}^{-\sum i_{j}} \operatorname{ch}_{z\left(\tilde{\pi} ; i_{1}, \ldots, i_{k-1}\right)}\right) \bullet\left(\frac{1}{d^{l}} \sum_{i_{k-l+1}, \ldots, i_{k}=0}^{d-1} \varepsilon_{d}^{-\sum i_{j}} \operatorname{ch}_{z\left(\sigma ; i_{k-l+1}, \ldots, i_{k}\right)}\right) \\
& =c\left(\frac{1}{d^{z-1}} M_{d}(z(\tilde{\pi})) \bullet \frac{1}{d} M_{d}(z(\sigma))\right)=c\left(\frac{1}{d^{z}} M_{d}(z(\pi))\right) .
\end{aligned}
$$

This completes the proof of $(*)$.

The inverse image of $\lambda_{d^{k}}$ under $c$ constructed in the preceding lemma may be simplified by means of a short analysis of the numbers $R(n, m)$. This will be done in three steps.

PROPOSITION 5.4. Let $n_{1}, n_{2}, m_{1}, m_{2} \in \mathbb{N}$ such that

$$
\operatorname{gcd}\left(n_{1}, n_{2}\right)=\operatorname{gcd}\left(m_{1}, m_{2}\right)=\operatorname{gcd}\left(n_{1}, m_{2}\right)=\operatorname{gcd}\left(n_{2}, m_{1}\right)=1 \text {. }
$$

Then we have $R\left(n_{1} n_{2}, m_{1} m_{2}\right)=R\left(n_{1}, m_{1}\right) R\left(n_{2}, m_{2}\right)$.

ProOF. By [4, Theorem 67], the Ramanujan sums have the following factorizing property: $\varrho\left(a_{1} a_{2}, b\right)=\varrho\left(a_{1}, b\right) \varrho\left(a_{2}, b\right)$ for all $a_{1}, a_{2}, b \in \mathbb{N}$ such that $\operatorname{gcd}\left(a_{1}, a_{2}\right)=1$. Furthermore, we have $\varrho\left(a, b_{1} b_{2}\right)=\varrho\left(a, b_{1}\right)$ for all $a, b_{1}, b_{2} \in \mathbb{N}$ such that $\left(a, b_{2}\right)=1$, 
as in this case taking the $b_{2}$-th power induces an automorphism of the group of $a$-th roots of unity. These two observations imply that

$$
\begin{aligned}
R\left(n_{1} n_{2}, m_{1} m_{2}\right) & =\sum_{x_{1} \mid m_{1}} \sum_{x_{2} \mid m_{2}} \varrho\left(n_{1} n_{2}, x_{1} x_{2}\right) \varrho\left(\frac{m_{1}}{x_{1}} \frac{m_{2}}{x_{2}}, 1\right) \\
& =\sum_{x_{1} \mid m_{1}} \sum_{x_{2} \mid m_{2}} \varrho\left(n_{1}, x_{1} x_{2}\right) \varrho\left(n_{2}, x_{1} x_{2}\right) \varrho\left(\frac{m_{1}}{x_{1}}, 1\right) \varrho\left(\frac{m_{2}}{x_{2}}, 1\right) \\
& =\sum_{x_{1} \mid m_{1}} \varrho\left(n_{1}, x_{1}\right) \varrho\left(\frac{m_{1}}{x_{1}}, 1\right) \sum_{x_{2} \mid m_{2}} \varrho\left(n_{2}, x_{2}\right) \varrho\left(\frac{m_{2}}{x_{2}}, 1\right) \\
& =R\left(n_{1}, m_{1}\right) R\left(n_{2}, m_{2}\right) .
\end{aligned}
$$

Let $\mathbb{P}$ be the set of all prime numbers.

PROPOSITION 5.5. For all $a, b \in \mathbb{N}_{0}$ and $p \in \mathbb{P}$, we have

$$
R\left(p^{a}, p^{b}\right)= \begin{cases}\mu\left(p^{a-b}\right) p^{b} & b \leq a ; \\ 0 & b>a .\end{cases}
$$

PROOF. For all $n, m \in \mathbb{N}$, the Ramanujan sum corresponding to $n$ and $m$ may be expressed in terms of the Möbius and the Euler function as follows:

$$
\varrho(n, m)=\mu(n / \operatorname{gcd}(n, m)) \frac{\varphi(n)}{\varphi(n / \operatorname{gcd}(n, m))}
$$

([4, Theorem 272]). Let $c:=\min \{a, b\}$ and $d:=\min \{a, b-1\}$. Then

$$
\begin{aligned}
R\left(p^{a}, p^{b}\right) & =\sum_{i=0}^{b} \varrho\left(p^{a}, p^{i}\right) \varrho\left(p^{b-i}, 1\right) \\
& =\varrho\left(p^{a}, p^{b}\right)-\varrho\left(p^{a}, p^{b-1}\right) \\
& =\mu\left(p^{a-c}\right) \frac{\varphi\left(p^{a}\right)}{\varphi\left(p^{a-c}\right)}-\mu\left(p^{a-d}\right) \frac{\varphi\left(p^{a}\right)}{\varphi\left(p^{a-d}\right)}
\end{aligned}
$$

and hence $R\left(p^{a}, p^{b}\right)=0$ for $b>a$, as $c=d=a$ in this case. Let $b \leq a$. Then we have $c=b$ and $d=b-1$, that is,

$$
R\left(p^{a}, p^{b}\right)=\mu\left(p^{a-b}\right) \frac{\varphi\left(p^{a}\right)}{\varphi\left(p^{a-b}\right)}-\mu\left(p^{a-b+1}\right) \frac{\varphi\left(p^{a}\right)}{\varphi\left(p^{a-b+1}\right)} .
$$

For $b<a-1$, this shows $R\left(p^{a}, p^{b}\right)=0$ as asserted. For $b=a-1$ it follows that $R\left(p^{a}, p^{b}\right)=-\varphi\left(p^{b+1}\right) / \varphi(p)=-p^{b}$, while, for $b=a$, we may conclude that $R\left(p^{a}, p^{b}\right)=\varphi\left(p^{b}\right)-\varphi\left(p^{b}\right) / \varphi(p)=p^{b}$. 
LEMMA 5.6. For all $n, m \in \mathbb{N}$, we have

$$
R(n, m)= \begin{cases}\mu(n / m) m & m \mid n \\ 0 & \text { otherwise. }\end{cases}
$$

PROof. Choose $a_{p}, b_{p} \in \mathbb{N}_{0}$ for all $p \in \mathbb{P}$ such that $n=\prod_{p \in \mathbb{P}} p^{a_{p}}$ and $m=\prod_{p \in \mathbb{P}} p^{b_{p}}$. Applying Propositions 5.4 and 5.5 we obtain

$$
\begin{aligned}
R(n, m) & =\prod_{p \in \mathbb{P}} R\left(p^{a_{p}}, p^{b_{p}}\right) \\
& = \begin{cases}\prod_{p \in \mathbb{P}} \mu\left(p^{a_{p}-b_{p}}\right) p^{b_{p}} & \forall p \in \mathbb{P}: b_{p} \leq a_{p} ; \\
0 & \text { otherwise }\end{cases} \\
& = \begin{cases}\mu(n / m) m & m \mid n ; \\
0 & \text { otherwise. }\end{cases}
\end{aligned}
$$

COROLlaRY 5.7. Let $d, k \in \mathbb{N}$. Then $M_{d}(k)=d \sum_{y \mid k} \mu(k / y) M_{d k, y}$.

PROOF. Let $y$ be a divisor of $d k$. Then Lemma 5.6 implies that

$$
R(d k / y, d)=\left\{\begin{array}{ll}
\mu(d k / d y) d & d \mid d k / y \\
0 & \text { otherwise }
\end{array}= \begin{cases}\mu(k / y) d & y \mid k \\
0 & \text { otherwise }\end{cases}\right.
$$

We are now in a position to give the proof of the Main Theorem 3.1.

Proof of the Main TheOREM 3.1. By Lemma 5.3 and (10), we have

$$
\left(\lambda_{d^{k}}, \zeta^{p}\right)_{S_{n}}=\frac{1}{k !} \sum_{\pi \in S_{k}} \frac{1}{d^{|z(\pi)|}}\left(M_{d}(z(\pi)), Z^{p}\right)
$$

But, for $\pi \in S_{k}$ and $q=q_{1} \ldots q_{k}:=z(\pi)$, we may conclude from Corollary 5.7 that

$$
\begin{aligned}
\frac{1}{d^{\mid z(\pi) !}}\left(M_{d}(z(\pi)), Z^{p}\right) & =\frac{1}{d^{k}}\left(M_{d}\left(q_{1}\right) \bullet \cdots \bullet M_{d}\left(q_{k}\right), Z^{p}\right) \\
& =\sum_{r_{1} \mid q_{1}} \cdots \sum_{r_{k} \mid q_{k}} \mu\left(q_{1} / r_{1}\right) \cdots \mu\left(q_{k} / r_{k}\right)\left(M_{d q_{1}, r_{1}} \bullet \cdots \bullet M_{d q_{k}, r_{k}}, Z^{p}\right) \\
& =\sum_{r \mid q} \mu(q / r)\left(M_{d q_{1}, r_{1}} \cdots \cdots M_{d q_{k}, r_{k}}, Z^{p}\right) .
\end{aligned}
$$

This completes the proof, as $\left(M_{d q_{1}, r_{1}} \bullet \cdots \bullet M_{d q_{k}, r_{k}}, Z^{p}\right)=\operatorname{syt}_{d * q, r}^{p}$ for all $r \mid q$, simply by definition of the scalar product $(\cdot, \cdot)$ and the convolution product $\bullet$ in $[6,1.3]$. 


\section{References}

[1] F. Bergeron, N. Bergeron and A. M. Garsia, 'Idempotents for the free Lie algebra and $q$ enumeration', in: Invariant theory and tableaux (Minneapolis, $M N$, 1988), IMA Vol. Math. Appl. 19 (Springer New York, 1990) pp. 166-190.

[2] D. Blessenohl and H. Laue, 'On the descending Loewy series of Solomon's descent algebra', J. Algebra 180 (1996), 698-724.

[3] A. M. Garsia and C. Reutenauer, 'A decomposition of Solomon's descent algebra', Adv. in Math. 77 (1989), 189-262.

[4] G. H. Hardy and E. M. Wright, An introduction to the theory of numbers, 4th Edition (Oxford University Press, Oxford, 1960).

[5] G. James and A. Kerber, The representation theory of the symmetric group (Addison-Wesley, Reading, Massachusetts, 1981).

[6] A. Jöllenbeck, 'Nichtkommutative Charaktertheorie der symmetrischen Gruppen', Bayr. Math. Schr. 56 (1999), 1-41.

[7] A. Jöllenbeck and M. Schocker, 'Cyclic characters of symmetric groups', J. Algebraic Combin. 12 (2000), 155-161.

[8] A. A. Klyachko, 'Lie elements in the tensor algebra', Siberian Math. J. 15 (1974), 914-920.

[9] W. Kraśkiewicz and J. Weyman, 'Algebra of invariants and the action of a Coxeter element', Bayr. Math. Schr. 63 (2001), 265-284.

[10] B. Leclerc, T. Scharf and J.-Y. Thibon, 'Noncommutative cyclic characters of symmetric groups', J. Combin. Theory Ser. A (I) 75 (1996), 55-69.

[11] F. Patras and C. Reutenauer, 'Higher Lie Idempotents', J. Algebra 222 (1999), 51-64.

[12] C. Reutenauer, Free Lie algebras, London Math. Soc. Monographs 7 (Oxford University Press, Oxford, 1993).

[13] I. Schur, Über eine Klasse von Matrizen, die sich einer gegebenen Matrix zuordnen lassen (Dissertation, Berlin, 1901).

[14] —_ 'Über die rationalen Darstellungen der allgemeinen linearen Gruppe', Sitzungsber. Pr. Akad. Wiss. (1927), 58-75.

[15] L. Solomon, 'A Mackey formula in the group ring of a Coxeter group', J. Algebra 41 (1976), $255-268$.

[16] R. Thrall, 'On symmetrized Kronecker powers and the structure of the free Lie ring', Amer. $J$. Math. 64 (1942), 371-388.

[17] E. Witt, 'Treue Darstellung Liescher Ringe', J. Reine Angew. Math. 177 (1937), 152-160.

Mathematical Institute

24-29 St Giles

Oxford OX1 3LB

UK

e-mail: schocker@maths.ox.ac.uk 